Supporting Information

\title{
Solving the "Magic Angle" Challenge in Determining Molecular Orientation Heterogeneity at Interfaces
}

\author{
Zhiguo Li, ${ }^{1}$ Jiaxi Wang, ${ }^{1}$ Yingmin $\mathrm{Li}^{2}{ }^{2}$ Wei Xiong**1,2
}

${ }^{1}$ Department of Chemistry and Biochemistry, University of California, San Diego, La Jolla, California 92093, United States

${ }^{2}$ Material Science and Engineering Program, University of California, San Diego, La Jolla, California 92093, United States

*Corresponding author. Email: w2xiong@ucsd.edu. Phone: 858-534-0270

Three Stretching Modes Related to Carbonyls in the Re-complex. The three stretching modes related to carbonyls in the $\mathrm{Re}(4,4$ '-dicyano-2,2'-bipyridine $)(\mathrm{CO})_{3} \mathrm{Cl}$ molecule are shown in Figure S1. They are labeled as A'(2), A" and A'(1) with center frequency of 1911, 1931 and $2020 \mathrm{~cm}^{-1}$ in 2D SFG spectra. $A^{\prime}(2)$ is an out-of-phase symmetric stretch, $A^{\prime \prime}$ is an anti-symmetric stretch and $A^{\prime}(1)$ is an in-phase symmetric stretch.$^{1,2}$ In the main manuscript we focus on analyzing $A^{\prime}(1)$ and $A^{\prime}(2)$ modes.

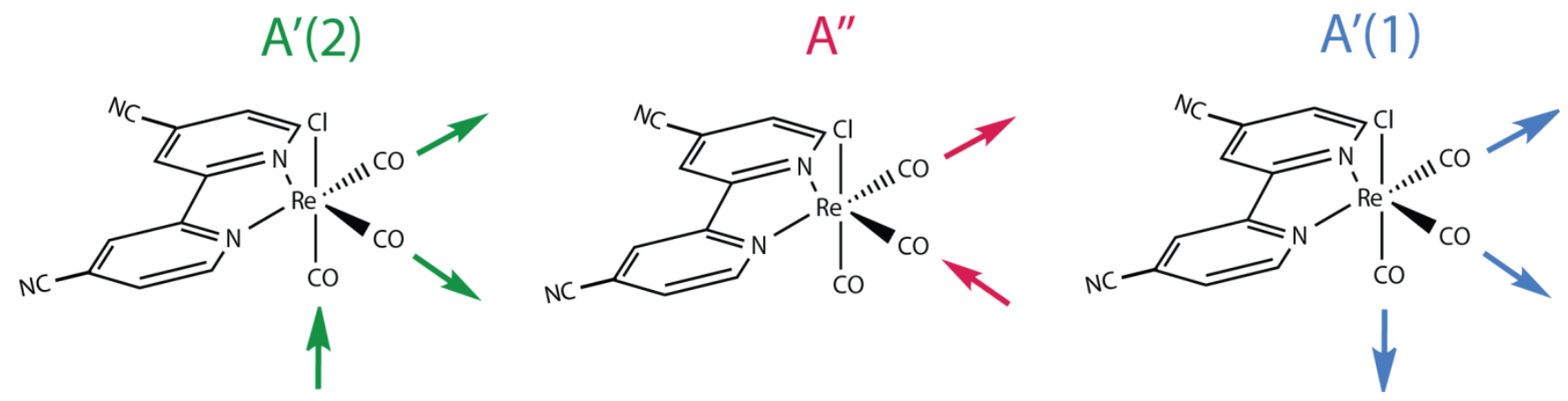

Figure S1. The three stretching modes related to carbonyls in the Re-complex.

Euler Transformation between Laboratory Coordinate Frame and Molecular Coordinate Frame. The three carbonyls in the Re-complex have an approximated $C_{3 v}$ symmetry, and the transition dipole moments of the three stretching modes are approximately perpendicular to each other. As shown in Figure S2, in the molecular coordinate frame, $z$ axis is defined along the transition dipole moment of A'(1) mode, which also coincides with the approximated $C_{3}$ symmetry axis; molecular $x$ and $y$ axes are defined along the transition dipole moments of $A^{\prime}(1)$ and $A^{\prime}$ ' modes, respectively. In the laboratory coordinate frame, $Z$ axis is defined along the surface normal and $X$ axis is in the incidence plane perpendicular to $Z$. The relationship between $X Y Z$ and $x y z$ frame are described by three angles: tilt angle $\theta$, in-plane rotation angle $\phi$ and twist angle $\varphi$.

In the SFG measurements, all beams were held at $p$ polarization. As the Fresnel factor is much larger in $Z$ direction compared with those in $X$ and $Y$ directions for all beams, both effective secondorder $\left(\chi_{\text {eff }}^{(2)}\right)$ and fourth-order $\left(\chi_{\text {eff }}^{(4)}\right)$ susceptibilities are dominated by singe susceptibility tensor element: 


$$
\begin{aligned}
& \chi_{\text {eff }}^{(2)} \approx L_{z Z}\left(\omega_{1}\right) L_{z Z}\left(\omega_{2}\right) L_{z Z}\left(\omega_{3}\right) \sin \beta_{1} \sin \beta_{2} \sin \beta_{3} \chi_{z Z Z}^{(2)} \\
& \chi_{\text {eff }}^{(4)} \approx L_{z Z}\left(\omega_{1}\right) L_{z Z}\left(\omega_{2}\right) L_{z Z}\left(\omega_{3}\right) L_{z Z}\left(\omega_{4}\right) L_{z Z}\left(\omega_{5}\right) \sin \beta_{1} \sin \beta_{2} \sin \beta_{3} \sin \beta_{4} \sin \beta_{5} \chi_{z Z z z z}^{(4)}
\end{aligned}
$$

where $L_{z Z}\left(\omega_{i}\right)$ and $\beta_{i}$ are the Fresnel factor and incidence/reflection angle of the $i^{\text {th }}$ beam. $\chi_{z z z}^{(2)}$ and $\chi_{\text {zzzzz }}^{(4)}$ are related to second-order $\left(\beta^{(2)}\right)$ and fourth-order $\left(\beta^{(4)}\right)$ molecular polarizabilities through the following equations:

$$
\begin{aligned}
& \chi_{Z Z Z}^{(2)}=N\left\langle\beta^{(2)}\right\rangle=N \sum_{i j k}\left\langle R_{Z i} R_{Z j} R_{Z k}\right\rangle \beta_{i j k}^{(2)} \\
& \chi_{Z Z Z Z Z Z}^{(4)}=N\left\langle\beta^{(4)}\right\rangle=N \sum_{i j k l m}\left\langle R_{Z i} R_{Z j} R_{Z k} R_{Z l} R_{Z m}\right\rangle \beta_{i j k l m}^{(4)}
\end{aligned}
$$

where $i, j, k, l, m=x, y, z ; N$ is the number density of Re-complex on the gold surface, bracket means taking orientationally averaged value, and $R$ is the element of Euler transformation matrix based on the geometry defined in Figure S2.

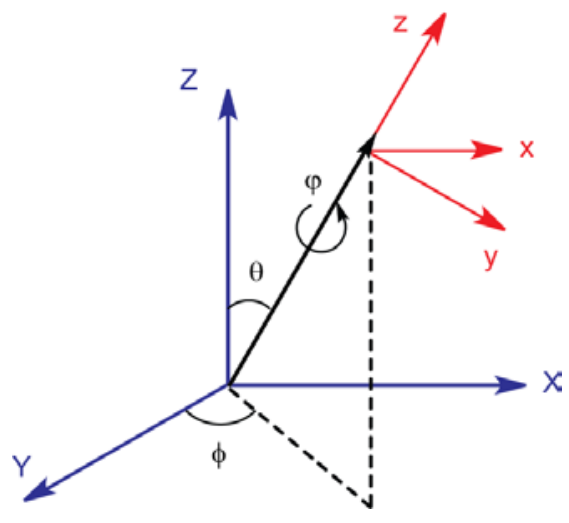

Figure S2. The relationship between $X Y Z$ frame and $x y z$ frame.

Assuming Re-complex has uniform distribution in the in-plane rotation angle $\phi$ and twist angle $\varphi$, Eq. S3-S4 can be simplified for mode $A^{\prime}(1)$ and $A^{\prime}(2)$, which are employed to determine the orientational parameters $D_{1}$ and $D_{2}$. For A'(1) mode,

$$
\begin{aligned}
& \chi_{z z z}^{(2)}\left[A^{\prime}(1)\right]=N\left[\left(\frac{\beta_{x x z}^{(2)}+\beta_{y y z}^{(2)}}{2}\right)\langle\cos \theta\rangle+\left(\beta_{z z z}^{(2)}-\frac{\beta_{x x z}^{(2)}+\beta_{y y z}^{(2)}}{2}\right)\left\langle\cos ^{3} \theta\right\rangle\right] \\
& \chi_{z z z z z}^{(4)}\left[A^{\prime}(1)\right]=N\left[\left(\frac{\beta_{x x z z z}^{(4)}+\beta_{y y z z z}^{(4)}}{2}\right)\left\langle\cos ^{3} \theta\right\rangle+\left(\beta_{z z z z z}^{(4)}-\frac{\beta_{x x z z}^{(4)}+\beta_{y y z z}^{(4)}}{2}\right)\left\langle\cos ^{5} \theta\right\rangle\right]
\end{aligned}
$$

For A'(2) mode,

$$
\left.\chi_{z Z Z}^{(2)}\left[A^{\prime}(2)\right]=N \mid\left(\beta_{x z z}^{(2)}\right)\langle\cos \theta\rangle+\left(-\beta_{x z x}^{(2)}\right)\left\langle\cos ^{3} \theta\right\rangle\right]
$$




$$
\chi_{z z z z z}^{(4)}\left[A^{\prime}(2)\right]=N\left[\left(\frac{3 \beta_{x z z x x}^{(4)}}{4}\right)\langle\cos \theta\rangle+\left(-\frac{3 \beta_{x z z x x}^{(4)}}{2}\right)\left\langle\cos ^{3} \theta\right\rangle+\left(\frac{3 \beta_{x z x x x}^{(4)}}{4}\right)\left\langle\cos ^{5} \theta\right\rangle\right]
$$

Modified Gaussian Distribution. A modified Gaussian function proposed by Simpson and Rowlen was employed to describe the distribution of molecular orientation. ${ }^{3}$ The representations are shown as follows:

$$
\begin{aligned}
f(\theta) & =\frac{1}{\sqrt{2 \pi} \sigma} e^{\frac{-\left(\theta-\theta_{0}\right)^{2}}{2 \sigma^{2}}} \\
f^{\prime}(\theta) & =\sum_{n=-4}^{4} f(2 \pi n+\theta)+f(2 \pi n-\theta)
\end{aligned}
$$

Where $\theta_{0}$ is the mean tilt angle and $\sigma$ describes the distribution width. The net distribution function $f^{\prime}(\theta)$, which is valid in the range from 0 to $\pi$, is developed from the normal Gaussian function $f(\theta)$. The values of $\langle\cos \theta\rangle,\left\langle\cos ^{3} \theta\right\rangle$ and $\left\langle\cos ^{5} \theta\right\rangle$ at different $\left(\theta_{0}, \sigma\right)$ pairs are calculated using Matlab through the following equation:

$$
\left\langle\cos ^{m} \theta\right\rangle=\int_{0}^{\pi} \cos ^{m} \theta \cdot f^{\prime}(\theta) \cdot \sin \theta d \theta
$$

Where $\sin \theta$ is a weighting function accounting for the fact that molecules may orient in both $X Z$ and YZ planes.

SFG Spectra Fitting. The 1D and 2D SFG spectra shown in Figure 3d (in main text) are fitted using the following equation: ${ }^{4}$

$$
\chi=\sum_{i=1}^{3} \frac{B_{i} \gamma_{i}}{\left(\omega-\omega_{i}\right)^{2}+\gamma_{i}^{2}}
$$

where $B_{i}, \omega_{i}$ and $\gamma_{i}$ represent the amplitude, center frequency and peak width of the $i^{\text {th }}$ vibrational mode, respectively. All the fitting parameters are shown in table S1. $\chi_{\text {eff }}^{(2)}\left[A^{\prime}(1)\right] / \chi_{\text {eff }}^{(2)}\left[A^{\prime}(2)\right]$ and $\chi_{\text {eff }}^{(4)}\left[A^{\prime}(1)\right] / \chi_{\text {eff }}^{(4)}\left[A^{\prime}(2)\right]$ are calculated using the following equation:

$$
\frac{\chi_{\text {eff }}\left[A^{\prime}(1)\right]}{\chi_{\text {eff }}\left[A^{\prime}(2)\right]}=\left(\frac{B\left[A^{\prime}(1)\right]}{\gamma\left[A^{\prime}(1)\right]}\right) /\left(\frac{B\left[A^{\prime}(2)\right]}{\gamma\left[A^{\prime}(2)\right]}\right)
$$

Table S1. Fitting Parameters for 1D and 2D Heterodyned SFG Spectra.

\begin{tabular}{l|lll}
\hline & A'$^{\prime}(2)$ & A $^{\prime}$ & A'(1) $^{\prime}$ \\
\hline
\end{tabular}




\begin{tabular}{c|ccccccccc}
\hline Mode & $B$ & $\omega$ & $\gamma$ & $B$ & $\omega$ & $\gamma$ & $B$ & $\omega$ & $\gamma$ \\
\hline 1D & $31.3(6)$ & 1912 & $52.8(7)$ & $11.3(4)$ & 1941 & $29.7(7)$ & $-48.1(5)$ & 2011 & $61.8(6)$ \\
2D & $2.5(2)$ & 1911 & $10.3(7)$ & $0.7(1)$ & 1931 & $9(2)$ & $-3.05(9)$ & 2020 & $9.7(4)$ \\
\hline
\end{tabular}

Calculation of Molecular Hyperpolarizability Tensor Elements. Energy and geometry optimization of Re-complex molecule were performed using B3LYP functional and LANL2TZ basis set with Gaussian 09. Dipole derivatives $\left(\partial \mu / \partial Q_{q}\right)$ and polarizability derivatives $\left(\partial \alpha / \partial Q_{q}\right)$ of each vibrational mode were obtained using the keyword "polar” and "iop(7/33=1)”. The $\partial \mu / \partial Q_{q}$ and $\partial \alpha / \partial Q_{q}$ values for A’(2) (mode 74 in Gaussian log file) and A’(1) (mode 76) modes are shown in Table S2.

Table S2. Dipole Derivatives $\left(\partial \mu / \partial Q_{q}\right)$ and Polarizability Derivatives $\left(\partial \alpha / \partial Q_{q}\right)$ for A'(1) and A'(2) Modes Determined from $a b$ initio Calculation.

Mode 74 (A'(2)):

Dipole derivatives: $3.07650 \mathrm{D}+01 \quad 3.89478 \mathrm{D}-03 \quad-3.04117 \mathrm{D}+00$

Polarizability derivatives:

$$
\begin{array}{ccc}
-0.988878 \mathrm{D}+00 & 0.505546 \mathrm{D}-03 & 0.241308 \mathrm{D}+01 \\
0.505546 \mathrm{D}-03 & 0.197090 \mathrm{D}+01 & -0.338132 \mathrm{D}-03 \\
0.241308 \mathrm{D}+01 & -0.338132 \mathrm{D}-03 & 0.228602 \mathrm{D}+01
\end{array}
$$

Mode 76 (A'(1)):

Dipole derivatives: $-4.24616 \mathrm{D}+00 \quad$ 1.33888D-03 $-3.61394 \mathrm{D}+01$

Polarizability derivatives:

$$
\begin{array}{ccc}
-0.970416 \mathrm{D}+00 & -0.419215 \mathrm{D}-04 & 0.117419 \mathrm{D}+01 \\
-0.419215 \mathrm{D}-04 & 0.141946 \mathrm{D}+01 & 0.173584 \mathrm{D}-03 \\
& & \\
0.117419 \mathrm{D}+01 & 0.173584 \mathrm{D}-03 & 0.440365 \mathrm{D}+01
\end{array}
$$

Measuring Depolarization Ratio Using Polarized Raman Spectroscopy. Polarized off-resonance Raman spectra were acquired with $647.09 \mathrm{~nm}\left(15,454 \mathrm{~cm}^{-1}\right)$ excitation, produced by a mixed krypton/argon gas-ion laser (Laser Innovations/Coherent; Innova 70C Spectrum). The wavelength was 
selected with an intracavity prism, and further isolated with a $647.1 \mathrm{~nm}$ bandpass filter (Semrock, MaxLine) placed in the beam path near the output of the laser. A right-angle geometry was used for the excitation and detection of scattered light. A spherical lens with focal length $80 \mathrm{~mm}$ was used to focus the laser beam into the bottom of a $1 \times 1 \mathrm{~cm}$ fluorescence cuvette that contained solvents for calibrations, or solutions of $\operatorname{Re}\left(4,4^{\prime}\right.$-dicyano-2,2'-bipyridine $)(\mathrm{CO})_{3} \mathrm{Cl}$. Raman scattering was collected and collimated with an F/1.2 camera lens (Canon FD $85 \mathrm{~mm}$ ). A doublet achromat with $300 \mathrm{~mm}$ focal length (CVIMelles Griot, 01LAO667) focused the light at the entrance slit of the spectrograph. Three optics were placed between the $300 \mathrm{~mm}$ lens and the entrance slit. First, a dichroic polarizer (OptoSigma, 0690120) mounted in a rotation stage was used to analyze the parallel (horizontally polarized) and perpendicular (vertically polarized) Raman scattered light. Second, a two-piece quartz wedge (OptoSigma, 068-6770) scrambled the polarization to compensate for different efficiencies of the spectrograph to parallel and perpendicular light. Third, a $647.1 \mathrm{~nm}$ long-pass filter (Semrock, RazorEdge) rejected the excitation line. The spectrograph (JY Horiba, iHR320) had a $320 \mathrm{~mm}$ focal length, and a single holographic grating with $1200 \mathrm{gr} / \mathrm{mm}$ groove density, $500 \mathrm{~nm}$ blaze. The detector was an open-electrode CCD (JY Horiba, Synapse). Raman shifts were calibrated based on six emission lines from a neon lamp (Oriel), and several Raman bands, ${ }^{6}$ from a 50:50 mixture of acetonitrile and toluene.

The rotation of the polarizer was optimized while collecting one-second spectra of carbon tetrachloride (Sigma Aldrich, $\geq 99.9 \%$ pure). The entrance slit of the spectrograph was set to $50 \mu \mathrm{m}$ (resolution $\sim 4 \mathrm{~cm}^{-1}$ ). After optimized positions were found, perpendicular and parallel spectra were recorded. The depolarization ratio of the $314 \mathrm{~cm}^{-1}$ and $459 \mathrm{~cm}^{-1}$ bands were determined by integrating the bands (perpendicular/parallel) and were 0.75 and $<0.01$, respectively. These depolarization ratios matched known values. ${ }^{7}$ Repeat measurements of $\mathrm{CCl}_{4}$ before and after collection of the rhenium samples yielded consistent depolarization ratios. Additionally, to check the performance of the system further in the red ( 720 nm), Raman spectra of benzene were collected and the $1585+1604 \mathrm{~cm}^{-1}$ bands were analyzed. This pair of bands had a depolarization ratio of 0.75 , as expected. ${ }^{6,7}$

The depolarization ratios of $\operatorname{Re}\left(4,4^{\prime}\right.$-dicyano-2,2'-bipyridine $)(\mathrm{CO})_{3} \mathrm{Cl}$ were recorded in DMSO solution at two concentrations, $2 \mathrm{mM}$ and $26 \mathrm{mM}$ (Table S3). The laser power at the sample was 45 $\mathrm{mW}$, and the entrance slit was $100 \mu \mathrm{m}$. Each polarization was collected for a total of $10-20$ minutes, and solvent-only spectra were also acquired for the same time. Spectra were processed using Igor Pro (WaveMetrics). Peaks caused by cosmic rays were removed manually from 1- or 2-minute acquisitions, and the acquisitions for the full collection time were then averaged. The solvent bands were subtracted from the spectra of $\operatorname{Re}(4,4$ '-dicyano-2,2'-bipyridine $)(\mathrm{CO})_{3} \mathrm{Cl}$ solutions by appropriate scaled subtraction (Figure S3). A broad luminescence background was removed from the solvent-free spectra using a spline fit. 


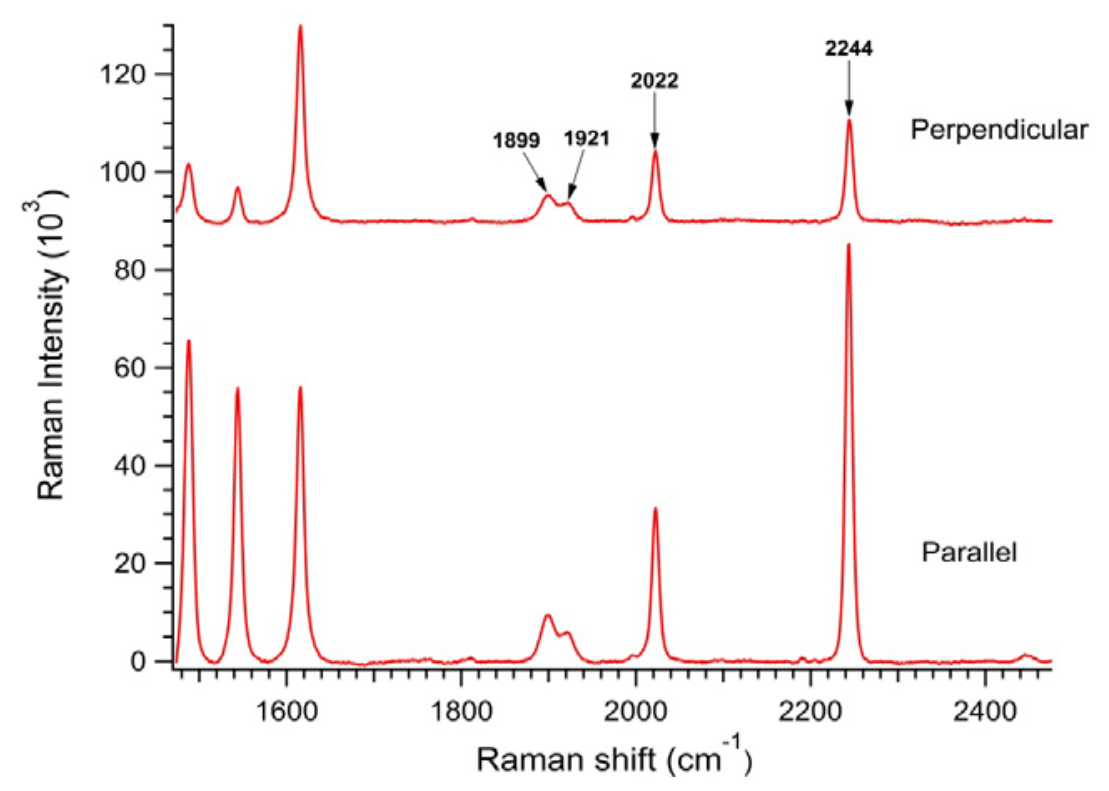

Figure S3. Polarized Raman spectra of Re(4,4'-dicyano-2,2'-bipyridine)(CO) ${ }_{3} \mathrm{Cl}$ in DMSO (26 mM) acquired with $647.09 \mathrm{~nm}$ excitation. Spectra are shown after subtraction of solvent bands and a broad luminescence background. The y-axis units are counts per 120-second acquisition time, and each of the two spectra are the average of 10 acquisitions (total collection time 20 minutes per spectrum). The perpendicular spectrum is offset $+90,000$ counts.

Table S3. Depolarization Ratios of Selected Bands of Re(4,4'-dicyano-2,2'-bipyridine)(CO) ${ }_{3} \mathrm{Cl}$ at Two Different Concentrations.*

\begin{tabular}{c|ccc}
\hline & A'(2) & A' & A'(1) \\
\hline $2 \mathrm{mM}$ & 0.55 & 0.58 & 0.44 \\
$26 \mathrm{mM}$ & 0.59 & 0.57 & 0.45 \\
\hline
\end{tabular}

* Values are determined by integrating the bands of polarized Raman spectra acquired with $647.09 \mathrm{~nm}$ excitation. We used the depolarization ratio of $2 \mathrm{mM}$ in the main manuscript. 


\section{Reference}

(1) Wang, J.; Clark, M. L.; Li, Y.; Kaslan, C. L.; Kubiak, C. P.; Xiong, W. Short-Range CatalystSurface Interactions Revealed by Heterodyne Two-Dimensional Sum Frequency Generation Spectroscopy. J. Phys. Chem. Lett. 2015, 6, 4204-4209.

(2) Clark, M. L.; Rudshteyn, B.; Ge, A.; Chabolla, S. A.; Machan, C. W.; Psciuk, B. T.; Song, J.; Canzi, G.; Lian, T.; Batista, V. S.; et al. Orientation of Cyano-Substituted Bipyridine Re(I) FacTricarbonyl Electrocatalysts Bound to Conducting Au Surfaces. J. Phys. Chem. C 2016, 120, 1657-1665.

(3) Simpson, G. J.; Rowlen, K. L. An SHG Magic Angle: Dependence of Second Harmonic Generation Orientation Measurements on the Width of the Orientation Distribution. J. Am. Chem. Soc. 1999, 121, 2635-2636.

(4) Shen, Y. R. Phase-Sensitive Sum-Frequency Spectroscopy. Annu. Rev. Phys. Chem. 2013, 64, 129-150.

(5) Boyd, R. W. Nonlinear Optics, 3rd ed.; Academic Press: Burlington, MA, 2008.

(6) McCreery, R. L. Raman Spectrosc. Chem. Anal.; John Wiley \& Sons, NY, 2000.

(7) Schrötter, H. W. Raman Spectroscopy with Laser Excitation. In Raman Spectroscopy: Theory and Practice; Szymanski, H. A., Ed.; Springer US: Boston, MA, 1970, 69-120. 